\title{
Palliative bladder radiotherapy: caveats in a changing landscape
}

\author{
Piet Dirix ${ }^{1,2}$, Michiel Strijbos $^{3}$, Tom Van den Mooter $^{3}$, Hendrik Vandeursen $^{4}$ \\ ${ }^{1}$ Department of Radiation Oncology, Iridium Netwerk, Oosterveldlaan 22, Wilrijk (Antwerp), Belgium; ${ }^{2}$ Translational Cancer Research Unit (TCRU), \\ Centre for Oncological Research (CORE), University of Antwerp, Edegem (Antwerp), Belgium; ${ }^{3}$ Department of Medical Oncology, GZA Sint- \\ Augustinus Hospital, Oosterveldlaan 24, Wilrijk (Antwerp), Belgium; ${ }^{4}$ Department of Urology, GZA Sint-Augustinus Hospital, Oosterveldlaan 24, \\ Wilrijk (Antwerp), Belgium \\ Correspondence to: Piet Dirix. Department of Radiation Oncology, Iridium Netwerk, Oosterveldlaan 22, 2610 Wilrijk (Antwerp), Belgium. \\ Email: Piet.Dirix@gza.be. \\ Provenance and Peer Review: This article is commissioned and reviewed by the Section Editor Dr. Xiao Li (Department of Urology, Jiangsu Cancer \\ Hospital, Jiangsu Institute of Cancer Research, Nanjing Medical University Affiliated Cancer Hospital, Nanjing, China). \\ Comment on: Ali A, Song YP, Mehta S, et al. Palliative Radiation Therapy in Bladder Cancer-Importance of Patient Selection: A Retrospective \\ Multicenter Study. Int J Radiat Oncol Biol Phys 2019;105:389-93.
}

Submitted Nov 14, 2019. Accepted for publication Feb 24, 2020.

doi: 10.21037/apm.2020.03.03

View this article at: http://dx.doi.org/10.21037/apm.2020.03.03

\section{Background}

Worldwide, bladder cancer is the ninth most common tumor (1). In muscle-invasive bladder cancer, the standard treatment consists of neoadjuvant cisplatin-based chemotherapy followed by radical cystectomy $(2,3)$. For patients who are considered unfit for surgery or who wish to attempt bladder-preservation, radiotherapy after maximal radical transurethral resection (TUR) represents a possible alternative. The addition of concomitant chemotherapy (whether mitomycine and 5-fluorouracil or cisplatin) to radiotherapy significantly improves outcome (4). With aggressive trimodality protocols (i.e., maximal TUR followed by concomitant chemoradiotherapy), survival rates similar those reported with radical cystectomy can be achieved (5).

In addition to its use in the curative setting, radiotherapy can provide considerable palliation in patients with metastatic disease or patients who are not candidates for definitive therapy. Prolonged hematuria is a frequent and debilitating complication in patients with bladder cancer (6). The hemostatic effect of radiotherapy is wellestablished and its therapeutic onset is generally observed very quickly (7). Several mechanisms contribute to the hemostatic effect of radiotherapy, including increased adhesion of thrombocytes to the vascular endothelium, blood vessel fibrosis and to some extent, tumor regression. In a non-curative setting, hypofractionation is usually preferred, as it is cheaper and provides greater comfort to patients by decreasing the time spent traveling. However, toxicity can be pronounced when conventional palliative schedules (using multiple daily fractions of 3-4 Gy) are prescribed. This is especially true for bladder radiotherapy, because several critical organs at risk (OAR) are located very close to the target (e.g., small bowel, rectum) and the bladder itself appears to be very sensitive to radiation. To minimize this toxicity, alternative fractionation schedules have been developed using weekly fractions (8). The major advantage of the longer delay between fractions is the potential recovery of acute toxicity. Still, palliative bladder radiotherapy remains a treatment with considerable sideeffects and patient selection is of the utmost importance. Clearly, physicians are not very good at estimating survival in metastatic cancer patients, and bladder cancer is a particularly aggressive disease (9).

Amin and colleagues performed a retrospective analysis from 2 British cancer centers (The Christie NHS Foundation Trust, Manchester and St James's University Hospital, Leeds) on 241 patients treated with palliative bladder radiotherapy (10). Interestingly, the indication for the bladder irradiation was local disease control (65\%) rather than hematuria (only 19\%). Still, $18 \%$ of patients had deceased within 30 days after RT and 14\% of patients could not even finish the planned radiation treatment. In all, one in four patients (25\%) underwent "futile" palliative bladder 
radiotherapy and were arguably unnecessarily treated. The authors offered little insights on how to improve patient selection, although they note that patients with good performance status and earlier stage disease survived longer.

First of all, the RT was truly palliative, using two opposing 2-dimensional, conventional anterior-posterior (AP) and posterior-anterior (PA) beams. This very basic techniques usually encompasses the target very broadly and allows little to no sparing of the surrounding OAR (e.g., rectum and small bowel in this case). Not surprisingly, $75 \%$ of patients reported symptoms at first follow-up. Also, there was a wide variety of fractionation schedules reported, ranging from a single fraction of 8.0 Gy (highly palliative) to 10 fractions of 3.0 Gy (probably more indicated to achieve long-term local control rather than short-term symptom palliation). A weekly schedule was only prescribed in about half of patients. In our center, we typically deliver palliative bladder radiotherapy for hematuria in a single fraction of 8.0 Gy using an AP-PA set-up. For local control we prescribe a weekly schedule over 6 weeks using modern volumetric arc therapy (VMAT), minimizing dose to the OAR. In both cases we perform on-line cone-beam CT imaging before treatment. In the current paper, the imaging protocol is unfortunately not described.

Secondly, patients were treated from 2014 to 2017, before the advent of immunotherapy revolutionized the systemic treatment of advanced bladder cancer. Up to 50 percent of patients are not candidates for cisplatin-based chemotherapy because of age or comorbidities (e.g., reduced renal function, neuropathy, heart failure). Although comparative phase III data are not available for first-line immunotherapy in this setting, two phase II studies supports its use and tolerability, with response rates between $20 \%$ to $30 \%$ and complete response rates around 10\% (11,12). Moreover, 5 antibodies (pembrolizumab, nivolumab, atezolizumab, durvalumab, and avelumab) targeting the programmed cell death-1 protein (PD-1) or its ligand (PD-L1) pathway have been approved by the US Food and Drug Administration (FDA) for patients who have progressed during or after platinum-based therapy (13). Again, responses to appear to be durable and are achieved in $17-26 \%$ of patients. Also, FDA recently granted accelerated approval for the fibroblast growth factor receptor (FGFR) inhibitor erdafitinib for patients with locally advanced or metastatic urothelial carcinoma who harbor a susceptible FGFR3 or FGFR2 genetic alteration and have progressed during or following platinum-containing chemotherapy (14). Consequently, patient selection for the right palliative
RT schedule (or none at all) will only become harder, since accurate prediction of patients who will respond to immunotherapy is still nearly impossible. Indeed, in patients treated with PD-L1 inhibitors for metastatic urothelial carcinoma, PD-L1 status is prognostic for objective response rate but not for overall survival and a predictive biomarker is still a long way off (15).

In conclusion, this retrospective analysis provides a worthwhile reminder to think twice before initiating palliative bladder radiotherapy, especially when using a longer fractionation schedule. There is absolutely no evidence that extremely hypofractionated radiotherapy, including single fraction RT, works less on symptom control than 5-10 fractions. Still, this historical cohort can only partially inform physicians in the current "immunotherapy era", where a (albeit limited) subset of patients prove to be long-term responders who could potentially benefit from local ablative treatment. A validated predictive biomarker of response to PD-1/PD-L1 inhibitors is yet to be defined, and this is perhaps the most significant unmet need in bladder cancer.

\section{Acknowledgments}

Funding: None.

\section{Footnote}

Conflicts of Interest: All authors have completed the ICMJE uniform disclosure form (available at http://dx.doi. org/10.21037/apm.2020.03.03). The authors have no conflicts of interest to declare.

Ethical Statement: The authors are accountable for all aspects of the work in ensuring that questions related to the accuracy or integrity of any part of the work are appropriately investigated and resolved.

Open Access Statement: This is an Open Access article distributed in accordance with the Creative Commons Attribution-NonCommercial-NoDerivs 4.0 International License (CC BY-NC-ND 4.0), which permits the noncommercial replication and distribution of the article with the strict proviso that no changes or edits are made and the original work is properly cited (including links to both the formal publication through the relevant DOI and the license). See: https://creativecommons.org/licenses/by-nc$\mathrm{nd} / 4.0 \%$. 


\section{References}

1. Ploeg M, Aben KK, Kiemeney LA. The present and future burden of urinary bladder cancer in the world. World J Urol 2009;27:289-93.

2. Bellmunt J, Orsola A, Leow JJ, et al. Bladder cancer: ESMO Practice Guidelines for diagnosis, treatment and follow-up. Ann Oncol 2014;25 Suppl 3:iii40-8.

3. Advanced Bladder Cancer Meta-analysis Collaboration. Neoadjuvant chemotherapy in invasive bladder cancer: a systematic review and meta-analysis. Lancet 2003;361:1927-34.

4. James ND, Hussain SA, Hall E, et al. Radiotherapy with or without chemotherapy in muscle-invasive bladder cancer. N Engl J Med 2012;366:1477-88.

5. Vashistha V, Wang H, Mazzone A, et al. Radical cystectomy compared to combined modality treatment for muscle-invasive bladder cancer: a systematic review and meta-analysis. Int J Radiat Oncol Biol Phys 2017;97:1002-20.

6. Abt D, Bywater M, Engeler DS, et al. Therapeutic options for intractable hematuria in advanced bladder cancer. Int J Urol 2013;20:651-60.

7. Cihoric N, Crowe S, Eychmüller S, et al. Clinically significant bleeding in incurable cancer patients: effectiveness of hemostatic radiotherapy. Radiat Oncol 2012;7:132.

8. Dirix P, Vingerhoedt S, Joniau S, et al. Hypofractionated palliative radiotherapy for bladder cancer. Support Care

Cite this article as: Dirix P, Strijbos M, Van den Mooter T, Vandeursen H. Palliative bladder radiotherapy: caveats in a changing landscape. Ann Palliat Med 2020;9(3):1269-1271. doi: 10.21037/apm.2020.03.03
Cancer 2016;24:181-6.

9. Bernson KR, Aggarwal S, Carter JN, et al. Predicting survival for patients with metastatic disease. Int J Radiat Oncol Biol Phys 2020;106:52-60.

10. Ali A, Song YP, Mehta S, et al. Palliative Radiation Therapy in Bladder Cancer-Importance of Patient Selection: A Retrospective Multicenter Study. Int J Radiat Oncol Biol Phys 2019;105:389-93.

11. Balar AV, Galsky MD, Rosenberg JE, et al. Atezolizumab as first-line treatment in cisplatinineligible patients with locally advanced and metastatic urothelial carcinoma: a single-arm, multicentre, phase 2 trial. Lancet 2017;389:67-76.

12. Balar AV, Castellano D, O'Donnell PH, et al. First-line pembrolizumab in cisplatin-ineligible patients with locally advanced and unresectable or metastatic urothelial cancer (KEYNOTE-052): a multicentre, single-arm, phase 2 study. Lancet Oncol 2017;18:1483-92.

13. Hussain SA, Birtle A, Crabb S, et al. From clinical trials to real-life clinical practice: the role of immunotherapy with PD-1/PD-L1 inhibitors in advanced urothelial carcinoma. Eur Urol Oncol 2018;1:486-500.

14. Loriot Y, Necchi A, Park SH, et al. Erdafitinib in Locally Advanced or Metastatic Urothelial Carcinoma. N Engl J Med 2019;381:338-48.

15. Ghate K, Amir E, Kuksis M, et al. PD-L1 expression and clinical outcomes in patients with advanced urothelial carcinoma treated with checkpoint inhibitors: a metaanalysis. Cancer Treat Rev 2019;76:51-6. 